\title{
Synergism and polymorphism in mixed surfactant systems
}

\author{
Ali Khan ${ }^{\mathrm{a}, *}$, Eduardo F. Marques ${ }^{\mathrm{a}, \mathrm{b}}$ \\ ${ }^{a}$ Physical Chemistry 1, Chemical Centre, Lund University, P.O. Box 124, SE-221 00 Lund, Sweden \\ bepartment of Chemistry, Coimbra University, 3049 Coimbra, Portugal
}

\begin{abstract}
Mixed surfactant systems have been, for a long time, one of the favorite areas for experimental studies on interfacial and bulk properties of surfactants. Beyond the well-known synergistic properties, with relevance to technical applications, recent studies increasingly focus on the bulk aggregation behavior. As more systematic and detailed experimental data is collected (for example, by use of scattering and direct imaging techniques), increasingly refined theoretical models are developed. Most references reviewed here clearly show both the trends. Topics such as micellar growth, micelle-to-vesicle transition and equilibrium vesicle formation in dilute systems (in particular in catanionic systems) continue to expand and sometimes pose challenges to conventional notions of surfactant self-organization. As the rich polymorphism of mixed aggregates is unraveled, the possibilities of using them for broader goals also increase (e.g. mesoporous materials and polymer-aggregate gels). (c) 2000 Elsevier Science Ltd. All rights reserved.
\end{abstract}

Keywords: Mixed surfactants; Self-assembly; Micelles; Vesicles; Gels; Mesoparticles

\section{Introduction}

When surfactants are added together in water, several physicochemical properties of the mixed system compared to those of the single surfactant system are changed due to the fact that there is a net interaction between the amphiphiles, i.e. due to non-ideal mixing. It is well known that many amphiphilic self-assemblies

\footnotetext{
Abbreviations: SDS, sodium dodecyl sulfate; DDAB, didodecyldimethylammonium bromide; $\mathrm{C}_{n} \mathrm{TAB}(\mathrm{C}), n$-alkyltrimethylammonium bromide(chloride); $\mathrm{C}_{16}$ PyC, cetylpyridinium chloride; $\mathrm{C}_{\mathrm{i}} \mathrm{E}_{\mathrm{j}}$, $j$-ethylene glycol mono-i-alkyl ether; POPC, 1-palmitoyl-2-oleoylsn-glycero-3-phosphocholine; POPG, 1-palmitoyl-2-oleoyl-snglycero-3-phosphoglycerol; cryo-TEM, cryo-transmission electron microscopy; SAXS, small-angle X-ray scattering; SANS, small-angle neutron scattering; SLS, static light scattering; DLS, dynamic light scattering

* Corresponding author. Tel.: +46-46-222-3247; fax: +46-46222-4413.

E-mail addresses: ali.khan@fkem1.lu.se (A. Khan), eduardo. marques@fkem1.lu.se (E.F. Marques).
}

and interfaces, biological or synthetic-based, consist of surfactant mixtures. The molecular structure of the surfactant (headgroups and tails), the presence of additives (salt, cosurfactant) and experimental variables ( $\mathrm{pH}$, temperature) can be manipulated in order to induce changes in interfacial activity and in intraand inter-aggregate forces. The mixed system almost invariably yields enhanced interfacial properties (e.g. decreased CMC, higher surface activity) with respect to the individual surfactants, in what is termed synergism. However, it is the rich polymorphism in bulk that has, in recent years, attracted increasing interest in experimental investigations and theoretical modeling.

This review deals with work in the field of mixed surfactant systems between 1998 to present date, including mostly non-ionic-ionic and cationic-anionic (catanionic) mixtures. Particularly active is the field of dilute catanionic systems, in which the strong interactions between headgroups, mediated by tail-tail interactions, leads to strong association and high degree of 
non-ideality. Often the two factors translate into a rich equilibria between different aggregated structures, such as non-classical micelles, equilibrium vesicles, several lyotropic liquid-crystalline phases and crystalline solids with thermotropic behavior.

\section{Mixed micelles}

\subsection{Catanionic systems}

It is now established that most of the mixed cationic-anionic surfactant systems, commonly known as catanionic systems, precipitate at equimolar concentrations at very high water content. At nonequimolar concentration, the mixture forms micelles with different sizes and shapes, closed bilayer vesicles and dilute lamellar phases (for a review on catanionic systems between 1943 and early 1996, see Khan and Marques [1]). The formation of aggregates and their transformation are rationalized in terms of interaction forces and surfactant geometry. These features are illustrated in the recently investigated system $\mathrm{C}_{16}$ TAB-SDS [2]. However, the cationic $\mathrm{C}_{16}$ TAB does not give any precipitation by mixing with $\mathrm{C}_{12} \mathrm{PO}_{3} \mathrm{Na}_{2}$ (disodium dodecane phosphonate) in water over a wide concentration range [3], showing that the ion-pair interaction is much weaker in this system compared to $\mathrm{C}_{16} \mathrm{TAB}-\mathrm{SDS}$ system. The plots of $\boldsymbol{\gamma}-\log C$ and $\Lambda_{\mathrm{d}}-C$, (where $\gamma$ is the surface tension, $\Lambda_{\mathrm{d}}$ is the differential conductivity and $C$ is the total concentration) detect four transitions for $C$ in the range $0.005-0.04 \mathrm{~mol} \mathrm{dm}^{-3}$. The first transition is ascribed to a monolayer condensation at the air/water interface, the second one, to ion-pair formation and the third, to CMC. The fourth transition, at concentrations higher than CMC is explained in terms of micellar growth, but the existence of vesicles cannot be excluded. The enhanced hydrolysis of the phosphate surfactant in the mixture also complicates the characterization of phase behavior. Interesting interfacial properties are also reported for the aqueous SDS $-\mathrm{C}_{16}$ PyC system [4]. At a total concentration of $1 \times 10^{-3} \mathrm{~mol} \mathrm{~kg}^{-1}$, the interfacial properties are reported to change abruptly at surfactant molar ratio $[\mathrm{SDS}] /\left[\mathrm{C}_{16} \mathrm{PyC}\right]=1: 3$ and 3:1. The observation is attributed to a closest hexagonal arrangement (due to strong ion-pair interactions) of the surfactant molecules at the air/water interface.

If, instead of anionic surfactant, a cationic one is added to another cationic surfactant, synergism is still observed. Addition of $\mathrm{C}_{6} \mathrm{TAB}$ to similarly charged $\mathrm{C}_{n} \mathrm{TAB}(n=8-16)$ decreases the CMC of the mixed systems, thus promoting micellization [5]. The molecular interaction parameter $\beta$ calculated from the regu- lar solution theory is found to become more negative with increased chain length.

\subsection{Ionic-non-ionic systems}

CMC is a fundamental physicochemical property of a surfactant, often determined by measuring the surface tension, $\gamma$, as a function of surfactant concentration, $C$. The presence of hydrophobic impurities in a surfactant is shown by a minimum in the $\gamma-\log C$ plot. In a recent study [6] comprising either single surfactants, mixed surfactants or surfactant admixed with another surface-active component, it is shown that the existence of a minimum or a negative peak in the $\gamma-\log C$ profile may imply that the amphiphile is a mixture, but the absence of the negative peak does not imply the absence of impurity or that the surfactant consists of only one component. The addition of very small amounts of the ionic SDS to the non-ionics Triton X-114 and Igepal CO-30 [7] causes a significant increase of the cloud temperature due to strong intermicellar repulsion. It is detected that within a range of solution composition, the clouding proceeds in distinct stages [7]. Prior to the conventional clouding, there is a colloidal phase also displaying a strong Tyndall scattering. Although this observation was first made in 1956 (see ref. 30 in McCarroll et al. [7]), the mechanism behind the existence of this preclouding state is yet to be understood.

Measurements on the fluorescence anisotropy of perylene indicate that the inclusion of SDS into Triton $\mathrm{X}-114$ micelles leads to a partial dislodging of perylene from the micellar palisade layer into the interior of the micellar aggregate [8]. Moreover, the monomers and aggregate species of the system are spectroscopically isolated and a hindered probe rotation is measured in the micellar environment. Mixtures of SDS with dodecylmalono-bis- $N$-methylglucomide (MEGA-12) [9] or with the sodium salt of $\alpha$-sulphonatomyristic acid methyl ester-decanoyl- $N$ methylglucomide (MEGA-10) [10] show non-ideal mixing in water, reflected in lower $\mathrm{CMCs}$ than those predicted for ideal mixtures. The formation of mixed micelles is particularly synergistic for non-ionic-rich compositions. However, the mixed micelle has a size and shape very similar to the two single surfactants and there is no local ordering within the mixed micelles. The mixture of the short chain cationic $\mathrm{NaC}_{6} \mathrm{SO}_{3}$ (sodium hexylsulphonate) and non-ionic $\mathrm{C}_{6} \mathrm{E}_{5}$ shows miscibility gap in water. At very low concentration, no aggregation behavior is observed for the single or the mixed systems, but small micellar aggregates are characterized for the mixture at high concentration [11]. The micellization process of the mixed sodium deoxycholate and non-ionic Tween 80 
[12] is comparable to that of the system SDS-non-ionic surfactant, namely in that the addition of the ionic surfactant decreases $\mathrm{CMC}$ and polar headgroup area and increases counterion binding. The aqueous solutions of the pairs $\mathrm{HCl}-\mathrm{C}_{8} \mathrm{E}_{4}$ and $\mathrm{NaCl}-\mathrm{C}_{8} \mathrm{E}_{4}$ are studied in the adsorbed film as well as in the micellar solution phase [13]. Analysis of the adsorbed film in equilibrium with bulk solution as well as the surfactant micelles in the presence of $\mathrm{HCl}$ and $\mathrm{NaCl}$, respectively, shows that the $\mathrm{Na}^{+}$but not the $\mathrm{Cl}^{-}$ion interacts electrostatically with the ethylene oxide group. This finding strongly suggests that the surfactant cation and not the counterion plays a critical role in the attractive interaction causing a deviation from ideal mixing for the mixed system $\mathrm{C}_{12} \mathrm{NH}_{4}^{+} \mathrm{Cl}^{-}-\mathrm{C}_{8} \mathrm{E}_{4}$ [13].

\section{Vesicle-forming systems}

The geometry of vesicles as closed bilayer aggregates of flexible colloidal size allows their use as in vitro cell models for biochemical studies, as agents for the transport of substrates (drug and gene delivery) and as nano-reactors for specialized chemistry, amongst other uses. There is a great demand for truly stable vesicle systems, strictly for thermodynamically stable vesicles. Since the early 1990s, experimental investigations on the phase behavior and aggregated structures of several mixed surfactant systems, in particular catanionic systems, have revealed that equilibrium vesicle formation is a relatively common phenomenon. Several theoretical models have also been put forth.

\subsection{Catanionic vesicles}

For mixtures of cationic and anionic surfactants, strong associative behavior occurs. A gradual addition of one of the surfactants to a solution of the other leads to formation and growth of mixed micelles, vesicle formation and eventually precipitation at equimolarity. The release of the counterions from the aggregate surface implies a high entropy of mixing, the major thermodynamic driving force for the association. The formation of a catanionic ion-pair (in presence of equimolar amount of salt) means that, in general, non-equimolar compositions are to be strictly treated as four-component systems, according to the phase rule. Thus, any ternary representation of phase behavior is only an approximation, often used for such pseudo-ternary systems.

For equimolar compositions, a crystalline lattice usually forms upon counterion release, as observed in several single-single-chained systems $[2,14,16,17]$ and single-double-chained systems $[18,19]$. If the solid is depleted from counterions a neutral swelling-type surfactant is obtained, catanionic surfactant [1]. A series of asymmetric catanionic surfactants have been synthesized and shown to yield rich thermotropic mesomorphism [20]. However, the 1:1 bulk composition may not always render insoluble catanionic pair, if the solid is not at the lowest energy state. This is the case for the system myristic acid $\left[\mathrm{CH}_{3}\left(\mathrm{CH}_{2}\right)_{12} \mathrm{COOH}\right]-$ cetyltrimethylammonium hydroxide $\left(\mathrm{C}_{16} \mathrm{TAOH}\right)$, where the acid-base reaction between the two amphiphiles leads to the formation of closed, flattened bilayer aggregates (nanodiscs) of micrometer size [21]. Another example is shown by the equimolar system $\mathrm{C}_{12} \mathrm{TAB}$-sodium para-toluene sulfate (SpTS), in which worm-like micelles are detected [22]. When the amphiphilic anion in $\mathrm{C}_{16}$ TApTS is replaced with sodium dodecylbenzensulfonate (SDBS) or SDS, the 1:1 catanionic pair is no longer soluble. Furthermore, in non-equimolar mixtures of $\mathrm{C}_{16}$ TApTS-SDBS vesicles are formed, whereas they are absent in the $\mathrm{C}_{16}$ TApDS-SDS system. Molecular modeling through the semi-empirical quantum-mechanical method, PM3, shows differences in chain packing for the ionpair between $\mathrm{C}_{16}$ TA:DS and $\mathrm{C}_{16}$ TA:DBS. In the mixture of $\mathrm{C}_{16} \mathrm{TAOH}$ with 3-hydroxy-2-naphtoic acid (HNC), a lamellar phase built up by stacked planar bilayers forms at 1:1 ratio [22]. If $\mathrm{HNC}$ is replaced with the corresponding sodium salt, densely packed onion-like vesicles are formed [23]. These studies globally illustrate that a suitable manipulation of headgroup and tail interactions can be done in order to design a specific type of aggregation feature. If the tail-tail interactions are made weaker (short alkyl chains, aromatic rings instead of alkyl chains), the catanionic may not precipitate and instead large micelles or vesicles are formed.

For mixtures with slight excess of the anionic or cationic surfactant, the precipitate is usually in equilibrium with vesicles at low surfactant volume fraction; and with dilute lamellar phases, at high volume fraction. In most systems, a region with only vesicles is observed. Detailed phase diagram studies illustrate these observations $[14,16,18,19]$. The presence of excess ionic surfactant is thought to break the symmetry of the monolayers and through different mechanisms (of entropic or energetic origin) to stabilize the vesicle bilayer with respect to competing geometries, in particular the planar bilayer. In general, the vesicles have properties that correlate with thermodynamic stability and show a relatively high degree of polydispersity (from tens of nanometers to a few micrometers). In the system SDS-DDAB, the average vesicle size and the presence of large micrometer-sized vesicles in the anionic- and cationic-rich side are detected by pulsed field gradient NMR self-diffusion for water $[18,19]$. In the cationic-rich area, a non-monotonic variation of 
vesicle size upon addition of SDS occurs, indicating the complex interplay between geometric packing and electrostatic interactions [19]. The dimensions of aggregate core and headgroup regions in mixed micelles and vesicles for the system $\mathrm{C}_{16} \mathrm{TAB}$-sodium octyl sulfate (SOS) are obtained from SAXS and SANS data [24]. Comparisons with the predicted values from thermodynamic cell models, seem to suggest that outof-phase fluctuations of headgroups occur in the catanionic interface, an effect that has to be taken into account in the free energy calculations for vesicle formation.

The possibility of catanionic vesicle formation in non-aqueous polar solvents has also been investigated. The phase behavior of equimolar mixtures of sodium alkylcarboxylates and alkyltrimethylammonium bromides in alcoholic solvents (ethanol, 1propanol and iso-propanol), dimethylsulfoxide (DMSO)-water and formamide-water mixed solvents has been studied [25]. While electron microscopy imaging and DSC methods may suggest aggregate formation in alcohols, alcohol mixtures and DMSO-water mixture (but not in the formamidewater mixed solvent), in another study electrical conductivity, SLS and DSC measurements failed to produce evidence for vesicle formation in the system $\mathrm{C}_{12} \mathrm{TAB}$-sodium dodecanoate in neat ethanol [26].

\subsection{Other vesicle-forming systems}

Formation of equilibrium vesicles can also occur in mixed systems other than the catanionic type, as shown by studies on systems of the type ionic surfactant-cosurfactant and weakly charged systems of the type non-ionic surfactant-ionic surfactant-cosurfactant-water (or brine). In the mixture formed by tetradecyldimethylaminoxide (TDMAO, a singlechained zwitterionic) and calcium dodecylsulfate (CDS), a birefringent lamellar phase composed of densely packed onion-like vesicles has been detected with an excess TDMAO, at constant $100 \mathrm{mM}$ of total amphiphile concentration [27]. In the mixed system formed by non-ionic surfactant (Triton X-100) and ionic surfactant $\left(\mathrm{C}_{16} \mathrm{PyC}\right)$ at low charge concentration, the addition of octanol can induce the formation of an equilibrium vesicle phase [28]. On the basis of SANS and direct cryo-TEM imaging fairly monodisperse vesicles with mean sizes much smaller (radii of the order of $8-10 \mathrm{~nm}$ ) than the usually observed ones are reported [28]. In the cationic-cationic system formed by DDAB- $\mathrm{C}_{12}$ TAC [29], in which the optimal chain packing determines aggregation behavior, vesicles are seen to form in the single-chain rich area of the phase diagram. One less conventional system where small monodisperse vesicles are suggested to form consists of the ternary system non-ionic surfac- tant $\mathrm{C}_{6} \mathrm{E}_{2}$-sodium cholate-water [30]. Upon addition of bile acid salt to the binary system $\mathrm{C}_{6} \mathrm{E}_{2}$-water, the miscibility gap is decreased. The vesicle size appears to increase as the temperature approaches the clouding point. Small monodisperse vesicles are also formed in the mixture formed by phospholipid POPC and sodium oleate/oleic acid and a so-called matrix effect is proposed to interpret the results [31].

\subsection{Micelle-to-vesicle transition}

The transition from micelles to vesicles, or viceversa, in surfactant mixtures, either by variation of the amphiphile mixing ratio or total volume fraction, has been a topic of long-standing interest. The original interest stems from the problem of protein reconstitution in lipid vesicles, whereby the latter are formed by surfactant depletion from mixed lipid-synthetic surfactant micelles. However, experimental studies have extended to include many surfactant mixtures of non-biological origin, in an attempt to model the effects of surfactant geometry and interaction forces. Recent studies have shown micellar shapes which go beyond the classical types, such as the spherical, rod-like and worm-like micelles. In catanionic systems, as the composition of the mixture is changed toward equimolarity, mixed micelles transform into vesicles. The shape and size of the mixed micelles and the thermodynamic nature of the transition vary with the characteristics of the system (geometry of surfactants, salt, temperature, etc.). The highly symmetric $\mathrm{C}_{12} \mathrm{TAB}-\mathrm{SDS}$ system has been re-investigated in ${ }^{2} \mathrm{H}_{2} \mathrm{O}$ in a series of papers, by SANS and SLS $\left[15^{\bullet}, 16\right]$. For a certain composition range, models for SANS data yield the presence of tablet-shaped monodisperse micelles (triaxial ellipsoids with axis $\mathrm{a}<\mathrm{b}<\mathrm{c}$ ), for both anionic- and cationic-rich areas. A first-order phase transition occurs from micelles to uni- or oligo-lamellar vesicles in coexistence with catanionic crystallites $\left[1^{\bullet}\right]$. Upon addition of $\mathrm{NaBr}$, the vesicles are either destabilized into lamellar sheets (closer to equimolarity) or into micelles. The micelles coexist with vesicles in a single thermodynamic phase for some compositions. Besides tablet-like micelles, self-avoiding worm-like ribbons with elliptical crosssection are also proposed, on the basis of SANS data. The salt-free $\mathrm{C}_{12} \mathrm{TAB}-\mathrm{SDS}$ vesicles are described within a SANS model of sticky hard spheres for the structure factor [16] and it is shown that upon increasing surfactant concentration, the vesicles transform into planar lamellar sheets. In the system SDSDDAB, addition of the double-chained amphiphile into SDS micelles induces moderate growth to globular micelles, initially, followed by a region of coexistence of micelle and small vesicles in the same phase [18]. The micelles change upon DDAB addition from 
globular to disk-like micelles, as imaged by the cryoTEM method. The influence of headgroup-tail interactions is demonstrated by the mixtures of $\mathrm{C}_{16} \mathrm{TAB}$ and aromatic-based headgroup amphiphiles [22]. The catanionic pair $\mathrm{C}_{16} \mathrm{TA}$ :pTS forms worm-like micelles, but addition of SDBS (i.e. replacement of the $\mathrm{CH}_{3}$ group by a dodecyl chain) induces vesicle formation.

The micelle-to-vesicle transition can also be induced by dilution. Surface tension studies by the drop volume method performed on the system $\mathrm{C}_{10} \mathrm{TAB}-$ SDeS show several break points in the $\gamma-\log C$, which are correlated with changes in aggregation behavior [17]. At several fixed values of the molar fraction of anionic surfactant in the mixture, three equilibrium regions seem to be identified in the $\gamma-\log C$ curve: (i) micelle-monomer; (ii) micelle-vesicle-monomer; and (iii) vesicle-monomer equilibrium. The experimentally obtained curves are in good qualitative agreement with a theoretical diagram based on a thermodynamic theory of micelle formation [17]. Another system in which a micelle-to-vesicle transition is induced by dilution is illustrated by the lecithin-bile salt system, which has received considerable attention throughout the years. A structural and kinetic study of the transition in the system egg yolk lecithinsodium taurochenodeoxycholate (STCDC) has been performed by combined SLS, DLS and SANS methods [32]. The transition from the polymer-like mixed micelles to vesicles, upon dilution, takes place via the formation of non-equilibrium disk-like structures. While the first step (worms $\rightarrow$ disks) is characterized by very fast kinetics, the second one (disks $\rightarrow$ vesicles) has a much slower rate constant. In the weakly charged system Triton X-100- $\mathrm{C}_{16} \mathrm{PyCl}$-octanol-water [28], it is observed that upon octanol addition, at fixed amount of charge, transitions from spherical micelles to worm-like micelles and, ultimately, to microvesicles are induced.

\section{Mixed aggregates in use}

\subsection{Mixed vesicles and synthetic macromolecules}

The interactions between vesicles based on mixed amphiphiles and polymers of synthetic nature, in particular polyelectrolytes, are of great interest. The use of vesicles coated with specifically designed watersoluble polymers can be of importance for drug delivery. The vesicle-polymer systems can also mimic to some extent the interactions between macromolecules and the cell membrane. In a few investigated systems, the interactions are especially pronounced since at least one of the vesicle components is oppositely charged to the polymer $\left[33^{\bullet}, 34-36^{\bullet}\right]$. The latter may consist of a homopolymer or a graft co-polymer with hydrophobic moieties. In the reviewed studies, the aim has been to investigate the effect of the macromolecule on the stability and shape of the vesicle membrane, or at the possibility of forming polymer-vesicle networks with rheological interest.

Net negatively charged vesicles prepared from the catanionic system SDS-DDAB have been mixed with cationic polyelectrolytes, either a homopolymer or a polymer modified with hydrophobic sites, where the

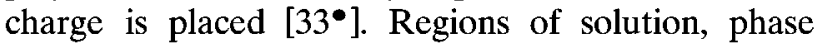
separation (with solid-like precipitate) and weak physical gel formation are identified in the phase maps. At low polymer concentrations, cryo-TEM imaging (Fig. 1) shows that the main effect of the homopolymer is to induce the formation of facet-shaped vesicles and, ultimately, disintegration of the vesicles into polydisperse disk-like structures. The hydrophobically modified polycation preferentially induces the formation of vesicle clusters, due to intervesicle bridging by the polymer chains $\left[33^{\bullet}\right]$. The formation of segregated domains in vesicles formed by mixed zwitterionic (POPC) and anionic (POPG) lipids, due to interaction with the polycation poly(vinylbenzyltrimethylammonium chloride) (PVTA), is demonstrated by ${ }^{2} \mathrm{H}$ quadrupolar splitting NMR [36 $6^{\bullet}$. The polycation is able to segregate laterally the anionic lipid into domains and, furthermore, it is demonstrated that the conformation of the adsorbed polymer into a collapsed or a random coil is dependent on the charge density of the bilayer. The anionic hydrophobically modified co-polymer PNIPAM-Py-Gly is seen to interact strongly with positively charged multilamellar vesicles formed by neutral lipids and cationic surfactants [35]. The interactions are dominated by the electrostatic attractions between the cationic lipid and the glycine residues of the polymer and, at high binding levels, regions of high local curvature are formed in the lipid bilayer. In mixtures of cardiolipin (an anionic lipid)-phosphatidylcholine vesicles and the cationic polyelectrolyte poly $(N$-ethyl-4-vinylpyridinium bromide (PEVP) [34], it is seen that the integrity of the vesicles is dependent on the mole fraction of negative charge in the mixed vesicle. Beyond a certain charge density of the vesicle, the polymer induces a disruption of the structure into non-vesicular PEVP-lipid complexes. Polycations adsorbed to negatively charged vesicles are shown to be desorbed by addition of anionic polyelectrolyte, which thus acts as a competitor with the vesicle aggregate [37]. If the polycation is made hydrophobically modified, the addition of the polyanion is much less effective in the desorption process. The thermosensitive anionic polymer PNIPAM-Py-Gly, also containing octadecyl sidechains, can bind to different types of mixed amphiphilic vesicles through electrostatic, hydrophobic and hydrogen-bonding interactions [38]. 


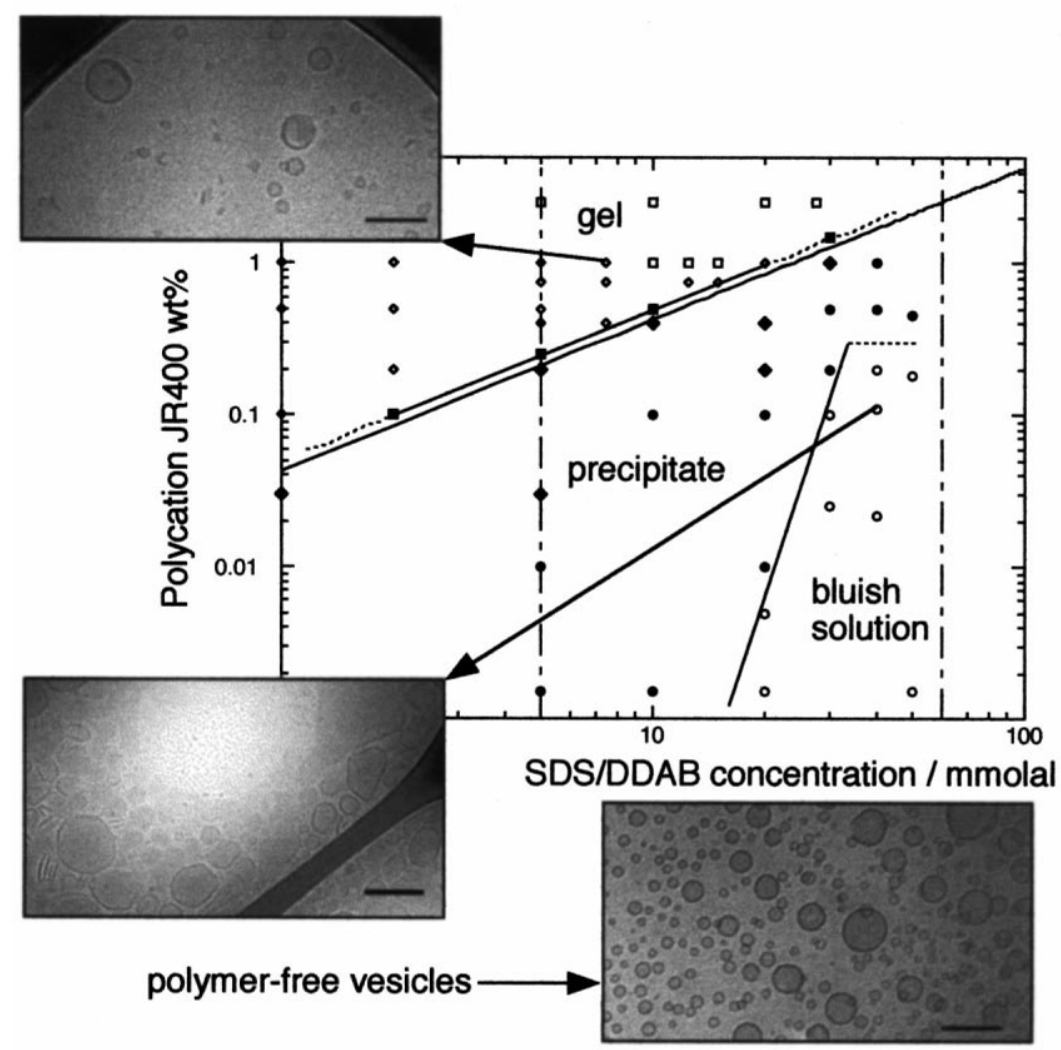

Fig. 1. Cryo-TEM images of faceted vesicles recorded in mixed solution and gel for the system polymer $\mathrm{JR} 400$-catanionic vesicles at $25^{\circ} \mathrm{C}$. Bar $=100 \mathrm{~nm}$ (for details, see Marques et al. [33॰]).

\subsection{Miscellaneous systems}

It is demonstrated that mixed surfactant aggregates are emerging as a new useful strategy for the synthesis of mesoporous particles [39]. The mixed systems also find their use in the determination of the dehydration coefficient of ethylene oxide groups of nonionic surfactants in mixed micelles [40]. An example of the potentialities of catanionic vesicles for technical applications is provided by the use of these aggregates in the electrokinetic chromatography (EKC) technique $\left[41^{\bullet}\right]$. It is demonstrated that the stable SDS- $\mathrm{C}_{12} \mathrm{TAB}$ catanionic vesicles have different selectivity and elution window from either SDS micelles or $\mathrm{SDS}-\mathrm{C}_{12} \mathrm{TAB}$ mixed micelles. Thus, catanionic vesicles have a great potential as aggregates for the pseudo-stationary phase in EKC.

\section{Phase behavior for concentrated systems}

The phase equilibria of mixed surfactant systems at high concentrations also exhibit interesting features which depend on the mixing ratio and self-assembly properties of the individual surfactants. Catanionic, cationic-non-ionic and cationic-cationic mixtures have been investigated at high surfactant concentrations. The triangular phase diagram for the catanionic system $\mathrm{C}_{12} \mathrm{TAOH}-\mathrm{C}_{12}$ PA shows a number of liquidcrystalline phases [42], in particular a cubic phase which is not present in the binary system. The addition of saccharose (LS), a neutral glycolipid, to the DDAB-rich dilute lamellar phases induces a decrease in the maximum swelling of that phase. At high dilution, it also induces a vesicle-to-micelle transition. The rationalization of these two facts is based on calculations of the osmotic pressure in lamellar and micellar phases through the Poisson-Boltzmann equation [43]. In another paper, the same authors investigate the phase behavior of the system DDAB-glycolipid, showing the existence of two distinct critical points, each for a different lamellarlamellar biphasic region, at room temperature [44 $\left.{ }^{\bullet}\right]$. A partial phase diagram is presented for the quaternary system $\mathrm{DDAB}-\mathrm{C}_{12} \mathrm{TAB}$-water-xylene, in which the volume fraction of the alkyl chain of the amphiphile is kept constant at 0.2 and the mixing ratio between surfactants is varied [45]. It is shown that upon oil addition to the $\mathrm{DDAB}-\mathrm{C}_{12} \mathrm{TAB}$-water mixture, micellar, lamellar, cubic liquid crystalline phases and micro-emulsions are formed. The lamellar phase region, formed at a high $\mathrm{DDAB} / \mathrm{C}_{12} \mathrm{TAB}$ ratio in the 
absence of oil, shifts to higher $\mathrm{C}_{12} \mathrm{TAB}$ content when xylene is added. The degree of oil penetration is enhanced with increasing $\mathrm{C}_{12} \mathrm{TAB}$ fraction and decreasing molecular size of the oil.

\section{Theoretical models}

Experimental findings in combination with theoretical predictions lead to a better understanding of the self-assembly processes of the mixed surfactant systems. Several models that have been published in the last 2 years are briefly reviewed here. Essentially, the models deal with the rationalization and prediction of mixed micelle properties (shape, size, CMC) or with the question of the thermodynamic stability of catanionic vesicles.

For systems consisting of mixed ionic-non-ionic surfactants, the composition of the ionic species in solution and aggregates can be accurately determined by ion selective electrodes. It is proposed that by using properties of cross-differentiation relations, the non-ionic surfactant activity can be obtained from variations of the ionic surfactant activity with different solution composition. This approach is used for the $\mathrm{C}_{12} \mathrm{TAB}-\mathrm{Brij} 35$ system and provides a description of the behavior of each solution component as a function of composition [46]. A detailed molecular thermodynamic model, in which the free energy of micellization is decomposed into five terms (transfer, interfacial, packing, steric and electrostatic contributions), is applied to predict experimental results concerning the systems SDS $-\mathrm{C}_{12} \mathrm{E}_{6}$ and $\mathrm{SDE}_{6} \mathrm{~S}-$ $\mathrm{C}_{12} \mathrm{E}_{6}\left[47^{\bullet}\right]$. The electrostatic and steric free energy terms play a major role for these mixed systems. The model is able to capture several features of the system such as the mixture CMCs, variation of micellar growth in the presence of electrolyte, hydration number, intermicellar interactions and other thermodynamic properties. Several experimental studies on catanionic micelles are also combined with theoretical modeling. A free energy expression for the formation of tablet-shaped micelles from the free monomers is presented, in terms of geometrical parameters (width and length of the aggregate) $\left[15^{\bullet}\right]$.

Based on droplet deformation and the van der Waals and long-range electrostatic interactions between droplets, an equation of state for osmotic pressure is proposed for micelles, emulsions and microemulsions [48]. The model is applied for the systems $\mathrm{C}_{12} \mathrm{E}_{5}-\mathrm{C}_{10} \mathrm{H}_{22}-\mathrm{H}_{2} \mathrm{O}$ and $\mathrm{C}_{12} \mathrm{E}_{5}-\mathrm{SDS}-\mathrm{C}_{10}$ $\mathrm{H}_{22}-\mathrm{H}_{2} \mathrm{O}$ and it not only has correlation ability but also good prediction capability. The deswelling of the lamellar phase formed in the water-rich part for the $\mathrm{DDAB}$, as well as the vesicle-to-micelle transition, on addition of an uncharged glycolipid is rationalized by osmotic pressure calculations based on PoissonBoltzmann equation [43].

In past years several models have been proposed to account for vesicle stability in catanionic systems, with respect to competing planar bilayer and micellar geometries (see for instance, refs [49-51]). A self-consistent microscopic model is developed to study the stability and phase equilibria of the catanionic vesicles [52]. Using a semiempirical Landau theory, the model shows that the catanionic mixture can stabilize spherical vesicles with respect to planar membranes. Furthermore, this model departs from a previous one by Safran and co-workers [49] in that it assumes that the physical basis for the breaking of the bilayer symmetry is interlayer interactions between the anionic and cationic molecules, rather than intralayer ones. Phase diagrams calculated are in qualitative agreement with experimental results on the previously studied SDBS-CTAT vesicles and are to reproduce non-monotonic effects on the vesicle radius along dilution paths observed for this system [52]. A model based on Monte Carlo simulations is also developed to study the influence of electrostatic interactions on the structure of the lipid headgroups in lipid-surfactant membranes [53]. In this model, the lipid molecules are treated as dipolar hard disks. The variation of ${ }^{2} \mathrm{H}-\mathrm{NMR}$ quadrupolar splittings measured at the $\alpha$ and $\beta-\mathrm{CH}_{2}$ segments of phospholipid in POPC $-\mathrm{C}_{12} \mathrm{E}_{4}$ mixtures are correlated with model prediction. The model is able to account for the experimentally observed effect of non-ionic surfactant in the lateral orientation order of the lipid headgroups [53].

\section{Conclusions}

The synergistic properties of mixed surfactant systems, of the type reviewed in this article, have been under investigation for the last five decades. However, with improved experimental techniques and more sophisticated modeling, the mixed systems are better characterized today. The discovery of stable vesicles and of the complex nature of the vesicle-to-micelle transition are of recent origin, drawing great interest to this area. Spontaneously formed vesicles (in the sense that they do not require strongly energetic methods to be formed) show many features that relate to thermodynamic stability. However, controversy between thermodynamically vs. kinetically stabilized vesicles still exists. More studies, especially of highly fundamental type, are essential to resolve the controversy. Mixed charged vesicles are shown to interact strongly with polyelectrolytes. Lateral domain formation in membranes, changes in vesicle shape and formation of networks are phenomena that have been reported, drawing increasing interest to such type of 
polymer-surfactant systems. Stable vesicles described in this review are mostly of synthetic origin. There is a great demand to search for environmentally friendly surfactants for the production of both stable vesicles and other surfactant aggregates.

\section{References and recommended reading}

- of special interest

$\bullet$ of outstanding interest

[1] Khan A, Marques E. In: Robb ID, editor. Catanionic surfactants in specialists surfactants. London: Blackie Academic and Professional (an imprint of Chapman \& Hall), 1997: 37-76.

[2] Tomasic V, Stefanic I, Filipovic-Vincekovic N. Adsorption, association and precipitation in hexadecyltrimethylammonium bromide/sodium dodecyl sulfate mixtures. Colloid Polym Sci 1999;277:153-163.

[3] Schulz PC, Minardi RM, Vuano B. Dodecyltrimethylammonium bromide-disodium dodecanephosphate mixed micelles. Colloid Polym Sci 1999;277:837-845.

[4] Patist A, Devi S, Shah DO. Importance of 1:3 molecular ratio on the interfacial properties of mixed surfactant systems. Langmuir 1999;15:7403-7405.

[5] López-Fontán JL, Suarez MJ, Mosquera V, Sarmiento F. Mixed micelles of $n$-alkyltrimthylammonium bromides: influence of alkyl chain length. Phys Chem Chem Phys 1999; 1:3583-3587.

[6] Lin S, Lin Y, Chen E, Hsu C, Kwan C. A study of the equilibrium surface tension and the critical micelle concentration of mixed surfactant solutions. Langmuir 1999;15: 4370-4376.

[7] McCarroll M, Toerne K, Wandruszka Rv. Micellar fluidity and preclouding in mixed surfactant systems. Langmuir 1998;14:2965-2969.

[8] McCarroll ME, Joly AG, Wang Z, Friedrich DM, Wandruszka Rv. Time-resolved fluorescence anisotropies in mixed surfactant solutions. J Colloid Interface Sci 1999;218:260-264.

[9] Griffiths PC, Whatton ML, Abbott RJ, Kwan W, Pitt AR, Howe AM, King SM, Heenan RK. Small-angle neutron scattering and fluorescence studies of mixed surfactants with dodecyl tails. J Colloid Interface Sci 1999;215:114-123.

[10] Okano T, Tamura T, Abe Y, Tsuchida T, Lee S, Sugihara G. Micellization and adsorbed film formation of a binary mixed system of anionic/nonionic surfactants. Langmuir 2000; $16: 1508-1514$

[11] Castaldi M, Constantino L, ortona O, Paduano L, Vitagliano V. Mutual diffusion measurements in a ternary system: ionic surfactant-nonionic surfactant-water at $25^{\circ} \mathrm{C}$. Langmuir 1998;14:5994-5998.

[12] Haque ME, Das AR, Moulik SP. Mixed micelles of sodium deoxycholate and polyoxyethylene sorbitan monooleate (Tween 80). J Colloid Interface Sci 1999;217:1-7.

[13] Matsubara H, Ohta A, Kameda M, Villeneuve M, Ikeda N, Aratono M. Interaction between ionic and nonionic surfactants in the adsorbed film and micelle: hydrochloric acid, sodium chloride, and tetrathylene glycol monooctyl ether. Langmuir 1999;15:54.

[14] Filipovic-Vincekovic N, Bujan M, Smit I, Tusek-Bozic L, Stefanic I. Phase transitions from catanionic salt to mixed cationic/anionic vesicles. J Colloid Interface Sci 1998; 201:59-70.

[15] Bergström M, Pedersen JS. A small-angle neutron scattering

- (SANS) study of tablet-shaped and ribbon-like micelles formed from mixtures of anionic and a cationic surfactant. $\mathbf{J}$ Phys Chem B 1999;103:8502-8513.
Non-classical micellar shapes for SDS-DTAB dilute mixtures, in the presence and absence of salt, are proposed on the basis of a SANS investigation. The dimensions for triaxial ellipsoids with half-axes $\mathrm{a}<\mathrm{b}<\mathrm{c}$, the tablet-shaped micelles, are calculated from the data. Addition of $0.1 \mathrm{M}$ induces a micellar growth to long flexible micelles with elliptical cross-section, the ribbon-like micelles.

[16] Bergström M, Pedersen JS, Schurtenberger P, Egelhaaf SU. Small-angle scattering neutron scattering (SANS) study of vesicles and lamellar sheets formed from mixtures of an anionic and a cationic surfactant. J Phys Chem B 1999; 103:9888-9897.

[17] Villeneuve $\mathbf{M}$, Kaneshina $\mathbf{S}$, Imae $\mathrm{T}$, Aratono $\mathbf{M}$. Vesicle-micelle equilibrium of anionic and cationic surfactant mixture studied by surface tension. Langmuir 1999;15:2029-2036.

[18] Marques EF, Regev O, Khan A, Miguel MG, Lindman B. Vesicle formation and general phase behavior in the catanionic mixture SDS-DDAB-water. The anionic-rich side. J Phys Chem B 1998;102:6746.

[19] Marques EF, Regev O, Khan A, Miguel MG, Lindman B. Vesicle formation and general phase behavior in the catanionic mixture SDS-DDAB-water. The cationic-rich side. J Phys Chem 1999;103:8353.

[20] Tomasic V, Popovic S, Filipovic-Vincekovic N. Solid state transitions of asymmetric catanionic surfactants. J Colloid Interface Sci 1999;215:280-289.

[21] Zemb T, Dubois M, Demé B, Gulik-Krzywicki T. Self-assembly of flat nanodiscs in salt-free catanionic surfactant solutions. Science 1999;283:816-819.

[22] Salkar RA, Mukesh D, Samant SD, Manohar C. Mechanism of micelle to vesicle transition in cationic-anionic surfactant mixtures. Langmuir 1998;14:3778-3782.

[23] Horbaschek K, Hoffmann H, Thunig C. Formation and properties of lamellar phases in systems of cationic surfactants and hydroxy-naphtoate. J Colloid Interface Sci 1998; 206:439-456.

[24] Iampietro DJ, Brasher LL, Kaler EW, Stradner A, Glatter O. Direct analysis of SANS and SAXS measurements of catanionic surfactant mixtures by Fourier transformation. J Phys Chem B 1998;102:3105-3113.

[25] Huang J-B, Zhu B-Y, Mao M, He P, Wang J, He X. Vesicle formation of 1:1 cationic and anionic surfactant mixtures in nonaqueous polar solvents. Colloid Polym Sci 1999;277: 354-360.

[26] Zana R, Michels B. On the formation of vesicles by mixtures of anionic and cationic surfactants in ethanol. Langmuir 1998; 14:6599-6602.

[27] Hoffmann H, Gräbner D, Hornfeck U, Platz G. Novel vesicles from single-chain surfactants. J Phys Chem B 1999; 103:611-614.

[28] Oberdisse J, Regev O, Porte G. Experimental study of the micelle-to-vesicle transition. J Phys Chem B 1998;102: 1102-1108.

[29] Viseu MI, Edwards K, Campos CS, Costa SM. Spontaneous vesicles formed in aqueous mixtures of two cationic amphiphiles. Langmuir 2000;16:2105-2114.

[30] Murthy K, Easwar N, Singer E. Spontaneous formation of monodisperse vesicles near the cloud point of an aqueous amphiphilic system. Colloid Polym Sci 1998;276:940-944.

[31] Lonchin S, Luisi PL, Walde P, Robinson BH. A matrix effect in mixed phospholipid/fatty acid vesicle formation. J Phys Chem B 1999;103:10910-10916.

[32] Egelhaaf SU, Schurtenberger P. Micelle-to-vesicle transition: a time-resolved structural study. Phys Rev Lett 1999;82: 2804-2807. 
[33] Marques EF, Regev O, Khan A, Miguel MG, Lindman B.

- Interactions between catanionic vesicles and oppositely charged polyelectrolytes - phase behavior and phase structure. Macromolecules 1999;32:6626.

Phase maps are presented for the polyelectrolyte-vesicle systems. Cryo-TEM imaging shows that the homopolymer JR400 induces faceted vesicles and disintegrations into disk-like structures, whereas the hydrophobically modified polymer LM200 promotes vesicle-vesicle bridging. Direct imaging of the physical gels obtained also show that the vesicle structure is preserved at high polymer content.

[34] Yaroslavov AA, Kiseliova EA, Udalykh OY, Kabanov VA. Integrity of mixed liposomes contacting a polycation depends on the negatively charged lipid content. Langmuir 1998; 14:5160-5163.

[35] Franzin CM, Macdonald PM, Polozova A, Winnik FM. Destabilization of cationic lipid vesicles by an anionic hydrophobically modified poly( $N$-isopropylacrylamide) copolymer: a solid-state ${ }^{31} \mathrm{P}$ NMR and ${ }^{2} \mathrm{H}$ NMR study. Biochim Biophys Acta 1998;1415:219-234.

[36] Crowell KJ, Macdonald PM. Surface charge dependence of

- polyelectrolyte-induced domain size and composition in lipid bilayer membranes. J Phys Chem B 1998;102:9091-9100.

Domain formation in a strongly interacting polymer-vesicle system is rigorously demonstrated by ${ }^{2} \mathrm{H}$ NMR. The quadrupolar splitting of choline-deuterated POPC is dependent in a known fashion on the surface charge density of the mixed vesicle. It is thus shown that the polycation adsorbs as a collapsed chain for higher charge density and in a more extended configuration for lower charge density.

[37] Yaroslavov AA, Koulkov VE, Yaroslavova EG, Ignatiev MO, Kabanov VA, Menger FM. Competitive interactions in negatively charged liposome-polycation-polyanion ternary systems. Langmuir 1998;14:5999-6004.

[38] Polozova A, Winnik FM. Contribution of hydrogen bonding to the association of liposomes and anionic hydrophobically modified poly ( $N$-isopropylacrylamide). Langmuir 1999;15: $4222-4229$.

[39] Ryoo R, Joo SH, Kim JM. Energetically favored formation of MCM-48 from cationic-neutral surfactant mixtures. J Phys Chem B 1999;103:7435-7440.

[40] Garamus V. A new method for the determination of the dehydration coefficient of ethylene oxide groups of non-ionic surfactants in mixed micelles. Chem Phys Lett 1998;290: 251-254.

[41] Hong M, Weekley BS, Grieb SJ, Foley JP. Electrokinetic

- chromatography using thermodynamically stable vesicles and mixed micelles formed from oppositely charged surfactants. Anal Chem 1998;70:1394-1403.
It is carefully demonstrated that the catanionic vesicles formed from the SDS-DTAB mixture can be favorably used as the pseudostationary phase in EKC. The vesicles show advantageous chromatographic properties, such as substrate selectivity, as compared to SDS micelles.

[42] Minardi RM, Schulz PC, Vuano B. The catanionic system dodecyltrimethylammonium hydroxide- $n$-dodecanephosphonic acid-water. Triangular phase diagram. Colloid Polym Sci 1998;276:589-594.

[43] Ricoul F, Dubois M, Zemb T, Plusquellec D. Micelle-smectic phase coexistence: origin of the maximum swelling of a mixed lamellar phase. Eur Phys J B 1998;4:333-340.

[44] Ricoul F, Dubois M, Belloni L, Zemb T. Phase equilibria and

- equation of state of a mixed cationic surfactant-glycolipid lamellar system. Langmuir 1998;14:2645-2655.

The equation of state relating osmotic pressure and composition for the mixed system is experimentally determined. The obtained phase diagram and the existence of a critical point for two biphasic lamellar-lamellar regions is discussed in terms of interaction forces beyond the DLVO theory.

[45] Aramaki K, Kunieda H. Solubilization of oil in mixed cationic liquid crystal. Colloid Polym Sci 1999;277:34-40.

[46] Palous JL, Turmine M, Letellier P. Mixtures of nonionic and ionic surfactants: determination of mixed micelle composition using cross-differentiation relations. J Phys Chem B 1998; 102:5886-5890.

[47] Shiloach A, Blankschtein D. Measurement and prediction of

- ionic/nonionic mixed micelle formation. Langmuir 1998; 14:7166-7182.

A comprehensive molecular-thermodynamic model for mixed micellization is developed. The model is able to successfully predict the micellar properties of two ionic-nonionic mixed systems, in which steric and electrostatic interactions are dominant.

[48] Li X-S, Lu J-F, Li Y-G, Liu J-C. A new molecular thermodynamic model for osmotic pressures in micelle and oil/water microemulsions systems with nonionic and ionic surfactants. Ind Eng Chem Res 1999;38:2817-2823.

[49] Safran SA, Pincus P, Andelman A, Mackintosh FC. Theory of spontaneous vesicle formation. Phys Rev 1991;43:1071.

[50] Bergström M. Thermodynamics of vesicle formation from a mixture of anionic and cationic surfactants. Langmuir 1996;12:2454. [51] Yuet PK, Blankschtein D. Molecular-thermodynamic modeling of mixed cationic/anionic vesicles. Langmuir 1996;12: 3802 .

[52] Duque D, Tarazona P, Chacón E. Microscopic model for mixed surfactant vesicles. Langmuir 1998:6827-6834.

[53] Schneider K-P, Eisenblätter S. Computer simulation of mixed lipid/non-ionic surfactant membranes. Chem Phys Lett 1999; 303:325-333. 